\title{
Park Pharmacy - A Modern Model for The In-Clinic Oncology Pharmacy
}

\author{
Stacey McCullough* \\ Department of Pharmacy, Tennessee Oncology, USA
}

Submission: July 20, 2018; Published: July 26, 2018

"Correspondence Address: Stacey McCullough, Senior Vice President, Pharmacy, Tennessee Oncology, USA, Email: smccullough@tnonc.com

\section{Opinion}

As an industry leader, Tennessee Oncology (TO) has achieved a decade of historical firsts. With a sustained drive and an innovative spirit, we're continually taking steps to improve patient experience. And, it's our patients who will inspire us to blaze new trails.

TO's long-standing vision of an in-clinic oncology pharmacy came to fruition in 2008 when we launched Park Pharmacy. Its mission is to provide patient-centered care through comprehensive services to help patients obtain orally administered specialty medications. To ensure patients achieve the best outcomes and receive seamless care, regardless of how the best treatment options are administered, Park Pharmacy focuses on maintaining the highest levels of customer service, responsiveness, accountability and integration into the broader healthcare team.

At the time, there were roughly a dozen oral treatment options on the market. Based on positive patient responses and treatment efficacy, they quickly became standard-of-care medications. They were practice changing and prompted a significant usage uptick. They also expanded oncology medication delivery to prescription benefits and dispensing pharmacies.

From the beginning, we carefully evaluated the pros and cons of oral therapy options in hematology/oncology. Oral options offer the convenience of taking medication at home without the disruption of frequent, and sometimes lengthy, clinic visits. But, self-care comes with increased responsibility. Patients and caregivers must learn how to take medications appropriately. Dosing schedules for medications that have weight-based dosing and individual pharmacokinetic medication profiles are frequently complex. Patients must have a full understanding of the number of pills they must take, when to take them, and whether this daily schedule varies within the monthly regimen cycle. It's this high-touch relationship that was the foundation for the "Specialty Pharmacy" niche industry concept.
To ensure success, we solicited expert advice from local, independent pharmacy owners. They offered guidance on licensing through the State Board of Pharmacy and on Park Pharmacy's daily operational management. In 2010, slightly over two years after we began pharmacy operations, we created the first PharmD role within the organization. Initially, its primary focus was two-fold: the intravenous drugs where the pipeline anticipated record number of approvals and simultaneously being a liaison between the practice and the retail pharmacy management group. But, as the specialty pharmacy space rapidly evolved, we recognized ancillary services that had been developed in the intravenous oncology space such as prior authorization and foundation assistance, were also paramount to the oral specialty pharmacy arena. Additionally, it was clear that electronic medical record (EMR) access and skilled staff with expertise in this area would be fundamental to Park Pharmacy's longevity.

We decided to internalize the pharmacy management. It was challenging, as we had to maintain cost neutrality while ensuring every aspect of service and execution surpassed previous experiences for both patients and providers. We implemented the transition in June 2011. Available oral medications were limited, so our top priority was capturing and filling every prescription written for any oral oncology product. Physicians weren't even aware that roughly 70 percent of oral medications were sent to and filled by outside specialty pharmacies. The goal promised two-fold results. Not only would we earn the confidence and trust of physicians and clinical staff, so they would send prescriptions to Park Pharmacy, but we would also gain access to insurer's specialty pharmacy networks to be able to process and fulfill prescriptions.

A retail group originally established Park Pharmacy as a retail pharmacy. Re-casting it as a specialty pharmacy meant establishing an operational framework and design necessary for call-center type phone reporting parameters, patient care plans, medication therapy management protocols and numerous operational and patient care reporting capabilities. Throughout the subsequent year, we created and documented general 
operating policies and procedures. We established telephonereporting capabilities of the average speed to answer calls and call abandonment. Our leaders paired workflow and technology to minimize error risk, and we trained pharmacy staff on clinical aspects of drug education capabilities.

During this time, the criteria for the specialty network was updated with a mandate for accreditation by the Utilization Review Accreditation Commission (URAC). At that time, few pharmacies achieved this distinction, but we embraced the daunting challenge. Not only was it a pre-requisite for network access, but we also believed earning accreditation would demonstrate the value of an internal pharmacy in the oncology arena.

Our commitment was unwavering. Together with a consultant firm, we conducted a mock staff survey, provided additional onsite training, and created quality improvement plans and quality monitoring. The requirements for accreditation became the daily standards-of-practice that ensured we met operations and patient care management quality standards and best-practice benchmarks.

Park Pharmacy prevailed, receiving its first accreditation from URAC in September 2013. Re-accreditation followed in 2016. Even though it took two years to achieve URAC accreditation, Park Pharmacy secured a second accreditation from the Accreditation Commission for Healthcare in 2016, as well, meeting the ever-higher access requirements of insurer networks. Over the next few years, our capture rate continued to incrementally increase. This slow growth demonstrated physicians were focused on patient care, and Park Pharmacy had to prove its competence and earn its place in collaborating on patient care. However, the broad requirements for all disease states that have available specialty medications continued to challenge the fulfillment rate.

Still, Park Pharmacy has fulfilled its initial goals of meeting patient needs and improving their experiences. Not only does the in-clinic pharmacy give patients a one-stop-shop for getting and filling prescriptions, but it also controls patient out-ofpocket expenses. Any out-of-pocket cost may be a deterrent to patient compliance and continued adherence. Therefore, we continually emphasize to our pharmacy staff that they ask, without exception, how the co-pay amount will impact the patient's finances.

Our staff members are also trained patient advocates. They regularly investigate the details of a patient's insurance benefits, help them with insurance form completion, and secure financial assistance when needed. In fact, in 2016, advocates secured $\$ 8$ million in patient assistance. Park Pharmacy also maintains and updates a working list of manufacturers co-pay cards. As a standard-of-practice, we proactively process co-pay cards for all qualifying patients. And, if a prescription mandates outside specialty pharmacy fulfillment, Park Pharmacy still ensures patient affordability.

To date, Park Pharmacy's impact has been dynamic. Inhouse coordination between providers and pharmacists has increased, and employing EMR access early in Park Pharmacy's development established a foundation for its long-term success. With immediate access to EMRs, it's easier to record and track patient medications and compliance and this shared information meets the level of collaboration needed to achieve best oral therapy outcomes.

We continue to explore new ways to create transparency for providers as the complex process of fulfilling a prescription for oral oncology medications progresses through the required steps. In addition, we're focused on increasing opportunities for patients to learn about therapeutics and their side effects and on engaging them in meaningful ways throughout their therapy to promote optimal adherence and persistency. The result will be better outcomes for a growing number of patients for whom oral therapies are the best, or only, treatment option.

Your next submission with Juniper Publishers will reach you the below assets

- Quality Editorial service

- Swift Peer Review

- Reprints availability

- E-prints Service

- Manuscript Podcast for convenient understanding

- Global attainment for your research

- Manuscript accessibility in different formats ( Pdf, E-pub, Full Text, Audio)

- Unceasing customer service

Track the below URL for one-step submission https://juniperpublishers.com/online-submission.php 